\title{
ZUM BEOWULF.
}

Beów. 219. Ymb ântîd òpres dôgores. Âutid, als 'eiue d. h. dieselbe zeit' gefasst und das von Heyne verglichenc bahuvrîli ânmôol decken sich nicht: ein subst. ânmồl grs. ânmôdes existiert nicht und nur dies könnte fitr ântîd in der bedeutung 'eine und dieselbe zeit' beweisend sein. Ich fasse antìd als partikelcomposition von and und tìd, eine dem ahd. antdag, antdago, mnl. andach ganz analoge bildung. Schon Ettmitiler hat in Veldeke's Eneide 168, 18 das wort ähnlich gedeutet, worauf Braune mich freundlichst aufmerksam macht: nur seine erklärung tempus constitutum, worauf ihn vielleicht as. êndago fuhrte, ist nicht untadelhaft. Andtag ist der 'gegentag', 'de dag die in dezelfde octaaf als gelyke wcekdag tegen den oorspronkelyken feestdag overstaat' (De Vries, Mnl. Wdb. 236), und ebenso antîd die gegenstunde, dieselbe stunde des folgenden tages. Die seefahrt dauerte also genau 24 stunden.

Beów. 901. Hì paes $\hat{a} r$ onঠâh. Niclits nötigt uns hier ein verbum onpeón anzunehmen; das von Heyne angefuihrte Otfridsche inthihan bringt kein licht. Ich schlage vor zu lesen: hè paes aron pâh, wie weordmyndlım pàh vs. 8. Dativi plur. auf -on sind anch in unserer dichtung nicht selten: scypon 1155, heáfdon 1243; an vielen stellen lïsst sich nicht entscheiden wie das auslautende $-\bar{u}$ aufzulösen ist, ob $-u m$ oder $-u n$. Im Orosius $\mathrm{L}$ ist das auslautende $-n$ im genannten casus ungemein häufig. Ein weiteres beispiel vs. 1933 unten.

Beów. 1118. Earme on eaxle ziehe ich zur vorhergehenden zeile und verstehe ich von den söhnen Hildeburhs, die 'den arm auf der schulter d. h. um den hals geschlagen', oder einander umhalsend auf den scheiterhaufen gelegt werden; 
nach eaxle setze ich also cin semicolon. Heyne thersah, dass nicht ron einem solne die rede ist, wie ausserdem der pluralis bânfatu (rs.1117) und vs. 107う̃ zur geniige beweisen. Sunu anstatt sun hat der uberarbeiter oder copist in seiner unschuld stchen lassen, wie nanche form mit eo fir ea u. s. w., welche ein nichtwestsächsisches original aufweisen.

lieów. 1201. Searonîðas fealh Eormenrices. Fealh soll nach Ettniuller subire, nach Grein subire, inire, nach Heyne (4. ausg.) 'gelangen, wozu konmen' bedeuten. Für die erste erklärung könnte man nl. velen anftihren, das wol dasselbe wort ist: es kommit nur vor in der rerbindung iets niet kunnen velen, etwas nicht leiden oder ertragen können, und gehört jetzt besonders der umgangsspraclie an. Was aber gegen die erklärung ron feolan als subire, inire spricht, ist der umstand, das sonst immer dies verbum intransitiv gebraucht wird: inne oder in feolan, on fleáme feolan, tô his ểle feolan, ofer mere feolan. Ich glaube daher, dass die vermutung Leos, welche Heyne in seiner ersten ausgabe aufuahm, später aber wider verwarf (vgl. nuch $\Lambda$ nz. fda. 3,180), hier das richtige trifft und dass wir es bloss mit einem schreibfehler zu tun haben: fealh für fleáh. Allerdings studigt dies gegen den grundsatz der textkritik, dass die lectio rarior den vorzug verdient; aber diesen verstoss kann man sich wol gefallen lassen, wenn man bedenkt, dass die verbinclung von fleón mit wörtern, welche feindschaft, hass, verfolgung, not und dergl. bedcuten, selır gewöhnlich ist: feóndscipas fleón (Beda III, 18), weón fleón (Gen. 1819, 2272), breá fleón (Gen. 2262), heleswengeas fleón (Beúw.) und weiter das bekannte foì her ôlachres nid. Weitor: setzt man nach sigle f. sincfel ein semicolon, wio die metrik und der zusammenhang zu fordern scheinen, 80 können die wörter searoniððas fecih $E$. schwerlich etwas anderes bedeuten als das ziemlich parallele geceás $\hat{e} c n e$ r $\hat{x} d$ und ist der sinn der dunklen stelle dieser, das Hâma's tod den nachstellungen Eormenrîces ein ende maclite. In Orosius Cott. V, 11, 1 findet sich umgekehrt fiéch statt fealh, was ich aber nicht als schreibfehler, sonder"I als absichtliche änderung betrachte, wie flugon statt fulgon in der Sachsenchronik $a^{0} 755$, Laud. MS. E.

Beów. 1215. Dass 'die halle lärm empfieng', wie Grein übersetzt, war gewiss höchst unpassend und störend, weil die 
königin im begriff war cine rede zu halten. Allerdings bedeutet swêg auch 'klang' (stefne swêg u. s. w.), aber der gedanke 'die halle empfieng klang' ist doch höchst sonderbar. Ich vermute healsbége onfêng; Weallhpeo fasste den halsring an, zeigte und reichte diesen dem Beowulf. Nur so laissen sich die worte brâc tisses beáges etc. recht begreifen. Für die bedeutung 'anfassen' verweise ich auf Gen. 2040: wếpna (acc. oder gen.?) onfôn, zu den waffen greifen.') Fur die construction verglciche man vs. 632: He pat ful gepeah ..., \& pâ gyddode gupe gefŷsed, Béóvulf mapelode etc.

Beów. 1248. Dic wörter an wîg gearwe, ad pugnam parati, taugen nicht in diesem zusammenhange: auch ist die alliteration hier vocalisch, nicht consonantisch. Man lese also andwiggearwe: sie waren immer bereit cinen überfall abzuschlagen, waren fortwithrend auf der but. Vielleicht aber ist nichts zu ändern, und steht anwîg flìr andwîg, wie sonst anstatt and-.

Beów. 1281. ĐÂr sôna wearð edhwyrft eorlum, sibðan etc. Heyne erklärt edhwyrft als 'widerkehr' sc. eines fruheren zustandes. Also 'der alte gefährliche zustand' muss binzugedacht werden, eine ellipse die immer etwas missliches hat. Ist vielleicht $\mathrm{zu}$ lesen sôra, gen. plur. v. sôr $=s \hat{a} r$, wie sôl vs. $302=s a l$, ôn vs. $2211=\hat{a} n$, unus? Auch die Cura Past. liat sôrig $227,8=$ sârig, die älteste chronik ôn $=\hat{a n} \mathrm{a}^{0} 879$; gleichfalls $m o \hat{n}=m \hat{a} n$ Fæder Lârcw. 82, Gnom Ex. 197; wîgrôd = wîgrâd Gen. 2084 .

Beów. 1301. In ar wird wol arn, das bekannte slêpern, stecken; folglich ist in in (h)im zu ändern, vgl. is fur his, C. P. $43,17$.

Beów. 1321. Aefter neodlaðu fasse ich als neád- oder nŷdlaðum und vergleiche nŷdbysgu. Heynes erklärung scheint mir sehr gezwungen. Neód und $n \hat{y} d$ werden bïufig verwechselt, und $e \dot{o}=e \dot{a}$ findet sich mehrfach. Jedenfalls ist neodladiu nicht richtig; ein dat. sg. auf $-u$ mit vernachlässigung des umlautes von $a$ ist unmöglich, denn diese endung kommt nur den abstractis zu, welche im gotischen auf $\hat{\imath}$ ausgehen.

1) Es liegt auf der hand, dass ags. onfôn 'empfangen' und onfón 'anfassen' nicht identisch sind: jenes ist mnl. ontfaen, dieses mnl. anevaen. 
Bców. 1347. Sele-rî́dende bedeutet nichts anders als boldagend 3113 ; was baben wir hier mit 'saalwächtern' zu tun? Es ist vollkommen parallel mit londbûend vs. 1346.

Bejw. 1364. Hrînde bearivas isî wol in hrîmde bearwas zu ändern = hrimige bearwas, wozu die reminiscenz in Blickling Hom. 209, $32 \mathrm{zu}$-vergleichen ist.

Beów. 1460. Ecg was îren, âtertânum fâh. Das von Bugge in seinem für die textkritik bahnbrechenden aufsatze (Tidskrift for Phil. VIII, 65) angefuhrte anord. eitrdropum fádr hat mich auf die vermutung gebracht, dass in der vorlage gestanden hat âtertêerum d. h. âterteárum fâh. Zu âterteár = atres teár vergleiche man huniges teár, Metr. XII, $10=$ huniteár (Leo, Bosw.), und balsames teár (Bosw.). Dass $r$ und $n$ häufig verwechselt, $\mathrm{ja}$ in vielen fällen nicht $\mathrm{zu}$ unterscheiden sind, ist hinlänglich bekannt: v\&. 1520 steht hord statt hond u. s. w.

Beów. 1605. Wiston \& ne wêndon bat hîe heora winedryhten selfne gesâwon. Wiston für ignorabant, wie Dietrich will, ist banal. Bugge deutet wie die englischen herausgeber (welche sich wenigstens durch dasjenige unterscheiden, was der Franzose irriger weise le sens commun nennt) hier wiston als wŷscton. Mit vollem rechte! Das $c$ wird lautgesetzlich im ags. (wie im mnl.) zwischen $s$ und $t$ syncopiert; freilich ist es durch systemzwang öfters wider eingefugt. Ein beispiel von wiste $=$ wysscte findet sich im Boethius cap. XXVI \&1 (in der ausgabe von Fox, seite 90): ne mê nếfie git ne lîcode eall pat ic wîsste. Der Cott. welcher die echt aelfredischen formen treuer bewalirt hat als die ziemlich schlechte handschrift, die Fox seiner textausgabe zu grunde gelegt hat, liest hier wisse! Wenn wir bloss uber den Cott. verfugen könnten, wurde gewiss die änderung von wisse in wyste höchst kühn sein!

Beów. 1785. Wîgeweorpad wird wol hier, wie El. 1196, in wigge geweorbad (El. 150) zu ändern sein: warum hat Heyne die formen mit igge $=\hat{\imath} g e$, wie z. b. vs. 1771, uberall aus dem text verbannt? Sind sie vielleicht nicht ags.? Diese spaltung des $\hat{\imath} g$ findet sich, wenigstens im ältesten westsächsischen, bloss vor folgendem $e$ : demnach habe ich seite 45 meiner kurzgefassten grammatik fälschlich stiggan angesetzt; man lese stigge (ascendat) oder stiggende. Wiggegeneorbad wird 
niggeweorpad geschrieben, wie firinon ondrŷsne, frinondrŷsne u. 8. w.

Beów. 1896. Weil sceáweras vs. 253 keine besucher oder touristen sondern spione sind (cf. Gen. 42,14 sceáveras, exploratores), so ist hier ein mutmassliches scâwan $=$ scâweras nicht am platze. Die handschrift (nach Wulcker) hindert nicht das 1804 vorkommende scapan hier einzusetzen. Auch vs. 243 vermute ich $\operatorname{sce}(a) \partial a n a=l a \partial \partial r a$ vs. 242.

Beów. 1933. Fremu folces cnên. Ich glaube nicht, dass wir Riegers fremu als framiô in sinne von 'fremd' annehmen düren: erstens ist das wort nirgends belegt, zweitens fordert die grammatik doppeltes $m$, drittens waren die königinnen naturlich fremde, viertens passt der begriff nicht in diesem zusammenhange. Auch Bugges 'strenua' (fremu anstatt freamu für freomu) will nir nicht gefallen, weil man hier keine löbliche eigenschaft erwartet, sondern eine solche die mit den wörtern môd firinon ondrŷsne in einklang steht. Ich halte den text fur verdorben; die heilung aber fur sehr einfach, wenn man nur das $m$ als ein verlesenes $c n$ der vorlage betrachtet: also frêcnu folces cnên, was einen trefflichen sinn gibt. Was firinon betrifft, siebe oben vs. 1785 und 901.

Beów. 2285. Onboren beága sum? was fehlte war bloss ein trinkgefäss.

Beów. 2339. Eallîrenne nîgbord im acc. neutr. ist nicht $\mathrm{zu}$ verdächtigen, obschon ein arger soloecismus. Bugge nimmt mit recht seine in 'Tidskr. for Philol.' vorgeschlagene einschaltung zurluck, bemerkt ebenfalls richtig, dass aucb anderswo (nur das citat aus Byrbtn. 146 beweist nichts, weil dort eben die bestimmte form sinngemãss ist) formen auf $e$ - vorkommen, wie z. b. êlterne, vergleicht aber, wie es mir vorkommt, mit unrecht lateinische zwillingswörter wie eburneus und eburnus. Es kann nicht zweifelhaft sein, dass sowol $\hat{c} t t e r n(e)$ als $\hat{\imath} \operatorname{sern}(e)=\hat{i}$ ren, isen mit dem suffix -in gebildet sind, aber schon frühe sind diese wörter nach dem muster von nesterne, cásterne und ähnlichen adjectiven auf gern. (r)ôni (lat. -âneus) mit anorganischem -e versehen. Beiläufig bemerke ich, dass auch wol got. Rumôneis demselben suffixe sein $\hat{o}$. verdankt: ich kann Kluge nicht beistimmen, wenn er zu beweisen sucht, dass lat. $\hat{a}$ zu germ. $\hat{o}$ geworden ist; das von ihm angefubrte pôl 
ist kein lelınwort, ebensuwenig wie nl. wörter pad, padde u. dergl.; und lat. palus 'morast' hat kur'zes $a: p \bar{a} l u s$ ist 'pfahl'.

Beów. 2374. Findan, impetrare. In dieser bedcutung liest man das wort auch in Beda IV, 9: Heó baed pat heó funde $\delta$ abôade oxl $\partial a s$ âr/asian Scyppendes mildheorlnysse bat heó fram swâ myclum cnylnnessum \& sna singâlum onlŷsed beón môste.

Beów. 2660. Ûrum bâm. Mit recht hält Heyne an der iiberlieferung fest: Bugges oder Riegers crgänzung bedurfen wir gar nicht. $\hat{U}$ rum bâm ist nicht zu verdächtigen; vergl.

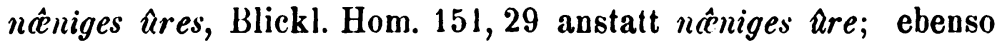
ûres nânes Cura Past. 211,14; urra selfra, 220,5; urne hnelcne 63,1. Wie unc und $\hat{u} s$ (z. b. Gen. 745 und 746) werden hier uncer und âre mit einander verwechselt. Auch das gotische scheidet den dual und plural nicht strenge.

Beów. 2905. Siexbennum seóc. Siexbennum crklärt Heyne wol richtig als seaxbennum; man vergleiche 2704 noelseaxe gebrấd. Ein ähnlicher ubergang von ea in ie findet sich in der Cura Past. 111, 23 forsieh in Hatton Ms., während die Cott. handschrift forseah (dcspexit) hat.

Beów. 2977. Die erklärung (hine) gewyrpan, aufspringen, sich erheben, entbehrt jeder autorität. Das wort bedeutet nur sich erholen, genesen. $\mathrm{Zu}$ den stellen in Greins glossar fuge ich noch hinzu Cura Past. 228, 2; Vita Guthlaci 86; und geedwyrped weorpan in Beda IV, 22, wo gleichfalls von einem vermundeten die rede ist, qui cum inter cadavera occisorum similis mortuo jaceret, landem recepto spiritu revixit.

Beów. 3063. Wundun hrdr, stolz auf scine wunden??? man vergleiche das sonderbare hwar, superbus, tumidus, Haupt, Ls. IX, 434. Wundun dat. plur. wie wîcun 1305 (wîcim, nach Wulcker, ist unglaublich).

Beów. 3115. Nâ sceal glêd frelan Weaxan wonna lêg nîgena strengel. Es liegt auf der hand, dass weaxan wonna lêg entweder verdorben ist oder eine andere bedeutung hat als man bisher angenommen hat. Heyne setzt die wörter in parenthesi, aber solche zwischenglieder bilden immer einen satz fur sich, wie hier nicht der fall ist, denn sceal muss aus dem vorhergehenden suppliert werden. Ich glaube, dass 
weaxan hier ein merkwurdiges synonymon ist von fretan oder peccean (letzteres gehört zu picgan und hat mit peccean 'decken' nichts zu tun; beispiele solcher verschiebung hat Paul VII, 134 gesammelt). Ein zweites beispiel von weaxan, fressen, verzehren, kenne ich nicht, weise aber auf das schon von Grein hervorgehobene merkwürdige waxgeorn, edax, in Aelfrici Colloquium (Thorpe, Anal.' 34): Leos erklärung 'vielfrässig, gernegross' befriedigt nicht. Ist vielleicht lat. vescor $\mathrm{zu}$ vergleichen?

LEIDEN, den 6. febr. 1882.

P. J. COSIJN.

\section{Berichtigungen.}

S. 359 z. 12 lies: H. Hund I, 52: peira er benlogum bregðd kunni.

S. $365^{2}$ lies: dem Norweger Eyvind. 
